\title{
Constraining the environment of neutrino-emitting regions in jets of FSRQs
}

\author{
Anita Reimer \\ Institute for Astro- \& Particle Physics, University of Innsbruck, 6020 Innsbruck, Austria \\ E-mail: anita.reimer@uibk.ac.at \\ Markus Böttcher \\ Centre for Space Research, North-West University, Potchefstroom, 2531, South Africa \\ E-mail: Markus.Bottcher@nwu.ac.za
}

\section{Sara Buson}

NASA Postdoctoral Program Fellow, Universities Space Research Association, USA

based at NASA Goddard Space Flight Center, Greenbelt, MD 20771, USA

now at University of Würzburg, 97074 Würzburg, Germany

E-mail: busoneastro.uni-wuerzburg. de

\section{Davide Miceli*}

University of Udine, DMIF, Udine, and

INFN, Trieste, Italy

E-mail: davide.miceli1993@gmail.com

\section{on behalf of the Fermi-LAT Collaboration}

\begin{abstract}
Recently, the gamma-ray blazar TXS $0506+056$ has been proposed as the source of neutrino events detected by IceCube in 2014-15 and 2017. This has further pushed the idea of blazar jets being among those cosmic sites where significant neutrino production may take place.

We use a procedure based on source-internal electromagnetic cascading to constrain the environments of neutrino-producing sites in photomeson production models of jetted AGN. The role of external photon fields as the dominant target for particle-photon interactions including possible effects of the anisotropic nature of the interactions in the co-moving jet frame is discussed.

We apply this procedure to the 2014-15 neutrino spectrum observed by IceCube from TXS 0506+056 to conduct a comprehensive study of these cascade spectra. By comparing these to the simultaneous multi-wavelength emission of this blazar constraints on the environment of the neutrino-emitting region are derived.
\end{abstract}

36th International Cosmic Ray Conference -ICRC2019-

July 24th - August 1st, 2019

Madison, WI, U.S.A.

\footnotetext{
* Speaker.
} 


\section{Introduction}

The recent detection of neutrinos from the direction of the blazar TXS 0506+056 [1, 2] has re-started a strong interest in considering beamed active galactic nuclei (AGN) as neutrino source candidates. Indeed, searches for the counterpart sources of the detected IceCube events have mostly been performed in the Fermi-LAT energy band (e.g., [2]) where blazars dominate the extragalactic sky implicitly assuming a causal connection between the neutrino and $\gamma$-ray emission.

TXS 0506+056, located at a redshift $z=0.3365[3,4]$ has recently been re-classified as "a masquerading BL Lac, i.e., intrinsically a flat-spectrum radio quasar with hidden broad lines and a standard accretion disk" [5]. With an estimated black hole mass of $\sim 3 \cdot 10^{8} \mathrm{M}_{\odot}$ [5] and corresponding Eddington luminosity $L_{\text {edd }} \sim 4 \cdot 10^{46} \mathrm{erg} / \mathrm{s}$ the available total jet power allows proton acceleration to ultra-relativistic energies. These relativistic hadrons interact in the highly radiative environment of the AGN, if above the threshold for photomeson and/or Bethe-Heitler pair production, to produce secondary particles, among them electron-positron pairs, neutrinos and high-energy photons. Dense target photon fields for particle-photon interactions of internal (i.e., jet radiation field) and/or external (e.g., accretion disk, broad-line region (BLR), etc) origin are required to assure a sufficiently high neutrino production rate $R_{\pi}$. Estimates for the BLR luminosity of TXS $0506+056$ lie at $L_{\mathrm{BLR}} \sim 5 \cdot 10^{43} \mathrm{erg} / \mathrm{s}$ [5], about $10 \%$ of its disk luminosity. On the other hand, dense photon fields prevent high-energy photon escape from its production region through $\gamma \gamma$-pair production. For a photomeson production optical depth $\tau_{\pi}=R^{\prime} R_{\pi} / c \gg 1$ (with $R^{\prime}$ the comoving size of the emission region) only photons of observed energy $\leq 10^{-5} E_{V \text {,obs }}$ (with $E_{v \text {,obs }}$ the observed neutrino energy) may escape the neutrino production site (see [6]).

On Sept, 22, 2017, TXS 0506+056 was found within the $50 \%$ containment region of the single IceCube event IceCube-EHE-170922A [1], and simultaneously in an elevated flux state in the Fermi-LAT energy range [1] at the time this event was detected. Archival searches for further neutrino events from this direction identified an neutrino excess (called "neutrino flare" in the following) consisting of $\sim 13$ neutrino events detected in a $110 \pm_{24}^{35}$ days period in September 2014 - March 2015 [2].

In this work we follow [6] and present a procedure that provides, in the framework of photohadronically produced neutrinos in jetted AGN, constraints on their associated broadband photon spectral energy distribution (SED) in a way that is independent of the origin of the dominant target photon field. Applying it to the neutrino flare of TXS 0506+056 together with its quasisimultaneous multi-wavelength (MWL) photon SED allows us to derive constraints on the origin of the target photon field. For this purpose we use the neutrino and photon MWL data as shown in [6].

\section{Theoretical setup and results}

Our theoretical setup is designed to derive the minimal requirements on the target photon field (of any origin) and the relativistic proton population to explain the observed neutrino (flare) flux and spectrum in a way that is as independent of model assumptions as is possible. The presented calculations consider the target photon field being isotropically distributed. In case of anisotropic particle-photon scatterings being important we do not expect significant modifications of the re- 
sulting spectra, as discussed below, however, changes of the required emission model parameters. This is relevant for external radiation fields providing the dominant target photons.

We first reconstruct, in the co-moving jet-frame, the minimum target photon spectrum required to produce the 2014 - 2015 neutrino flare spectrum, and calculate all corresponding further secondary particles produced through photomeson and Bethe-Heitler pair production in this radiation field (see Fig. 1). This fit determines a combination of parameters pertaining to the relativistic proton spectrum and the target photon field, leaving their relative normalization as a free parameter. Note that a given target photon field readily determines photomeson, Bethe-Heitler pair production, inverse Compton and $\gamma \gamma$ pair production interaction rates. A rather narrow energy range at hard X-rays (in the co-moving jet frame and, e.g., for a Doppler factor $D=10$ it ranges from $\left.\varepsilon^{\prime}=10-60 \mathrm{keV}\right)$ is found sufficient to explain the neutrino flare spectrum by photomeson production of relativistic protons from an injection spectrum with index $\alpha_{p}=2.0 \pm_{0.2}^{0.1}$ extending to $\mathrm{PeV}-$ hundreds of PeV energies. Fit solutions for Doppler factors $D=1-50$ have been found this way.

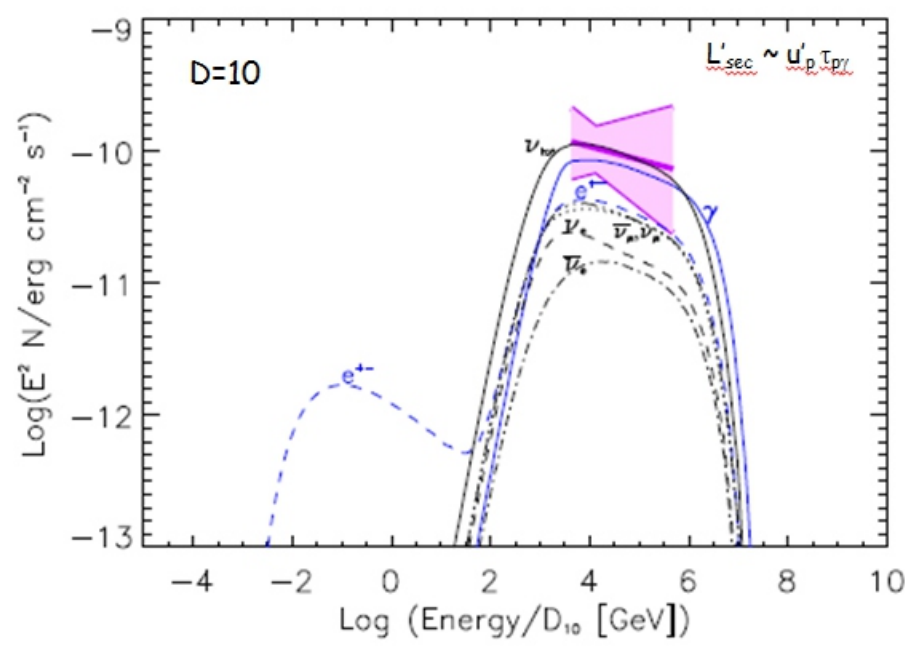

Figure 1: Secondary particles (muon neutrinos: dotted black lines, anti-muon neutrinos: dashed-triple dotted black lines, electron neutrinos: dashed black lines, antielectron neutrinos: dashed-dotted black lines, total neutrino yield: solid black line, electron-positrons: dashed blue line, photons: solid blue line) at production due to proton-photon interactions (photomeson and Bethe-Heitler pair production) on the same target photon field, in comparison to the total flux as measured by IceCube during the 2014 - 15 neutrino flare (violet bow tie), corrected for neutrino oscillations. Energies and fluxes are given in the observer frame. Neutron decay neutrinos are neglected. This figure is adapted from [6].

For external target radiation fields the angular distribution of the target photons in the comoving jet frame may become anisotropic and will lead to an increase or lowering of the particlephoton and photon-photon collision rates. Note that this affects photomeson, Bethe-Heitler pair, $\gamma \gamma$ pair production and inverse Compton interaction rates to the same amount as long as collisions occur in the same target photon field. The observed neutrino flux level would then be matched by adjusting the injected proton energy density correspondingly in the case of scatterings involving target photon populations that are anisotropic in the jet frame. The ratio of the secondary pair and photon number density to that of the neutrinos remains, however, constant.

The secondary particles from particle-photon interactions initiate pair cascades, to an amount that depends on the $\gamma \gamma$ opacity, in the emission region. A specific choice of proton power (and, thus, target photon energy density) determines the value of the $\gamma \gamma$ opacity of the neutrino production region. Depending then on the relative energy densities of the magnetic field $\left(u_{B}^{\prime}\right)$ and the target photon fields $\left(u_{t}^{\prime}\right)$, electromagnetic cascades (considered to be linear) develop in the synchrotron- 
$\left(u_{B}^{\prime} \gg u_{t}^{\prime}\right)$, synchrotron-Compton $\left(u_{B}^{\prime} \approx u_{t}^{\prime}\right)$, or Compton-dominated $\left(u_{B}^{\prime} \ll u_{t}^{\prime}\right)$ regimes.

If inverse Compton (IC) scattering supports the cascade $\left(u_{t}^{\prime} \gg u_{B}^{\prime}\right)$, the emerging photon spectra are fully determined once the opacity due to photon-photon pair production is fixed. This energy-dependent opacity in turn is related to the optical depth of photon-proton interactions, which itself is linked to the neutrino spectral flux. Compton-supported cascading on the minimal target photon field (as determined above) therefore provides the corresponding minimal cascade flux for $u_{t}^{\prime} \gg u_{B}^{\prime}$. In the following we choose to vary the maximum of the energy-dependent photon-photon opacity, $\tau_{\gamma \gamma \text {,max }}$ (which here serves as a proxy for $\left(R^{\prime} \cdot u_{t}^{\prime}\right)$ ) to calculate the corresponding cascade spectra. Absorption in the extragalactic background light (EBL) is taken into account using the model of [7]. For $\tau_{\gamma \gamma, \max } \ll 1$ we find internal absorption neglegible, and the emerging IC flux increases with increasing opacity (since $\tau_{\gamma \gamma} \propto \tau_{\text {IC }}$ ). None of these cascade spectra reach the flux level of the observed MWL (in particular, Fermi-LAT) data (see [6]). Therefore, in these cases, most of the photon flux has to be produced by emission processes occuring in the source that were not initiated by proton-photon interactions. When increasing the opacity further, $\tau_{\gamma \gamma} \gg 1$ (e.g., Fig. 2), deep absorption troughs in the GeV-range appear. Any photons in the $\mathrm{GeV}$ range produced cospatially to the neutrino flux, will inevitably suffer from internal absorption and will subsequently be cascaded to lower energies, independent of their production process. In this case of an efficient neutrino producer, the observed $\mathrm{GeV}$-flux has to be produced in a region that has no dense radiation fields at $\mathrm{keV}$ energies (i.e., different to the neutrino production site), either within the jet of TXS $0506+056$ or in a different location. As a consequence, there is no causal connection between the observed neutrino flux and observed GeV-flux.

The Compton optical depth $\tau_{\mathrm{IC}}$ may be altered when scatterings sample photon fields that are anisotropic in the co-moving frame. Since $\tau_{\mathrm{IC}}$ is directly linked to $\tau_{\gamma \gamma}$ and the emerging cascade spectra presented here are proxied by $\tau_{\gamma \gamma}$, no significant changes of the Compton cascade spectra for a given $\tau_{\gamma \gamma}$ are expected in such cases.

Figure 2: Compton-supported cascade

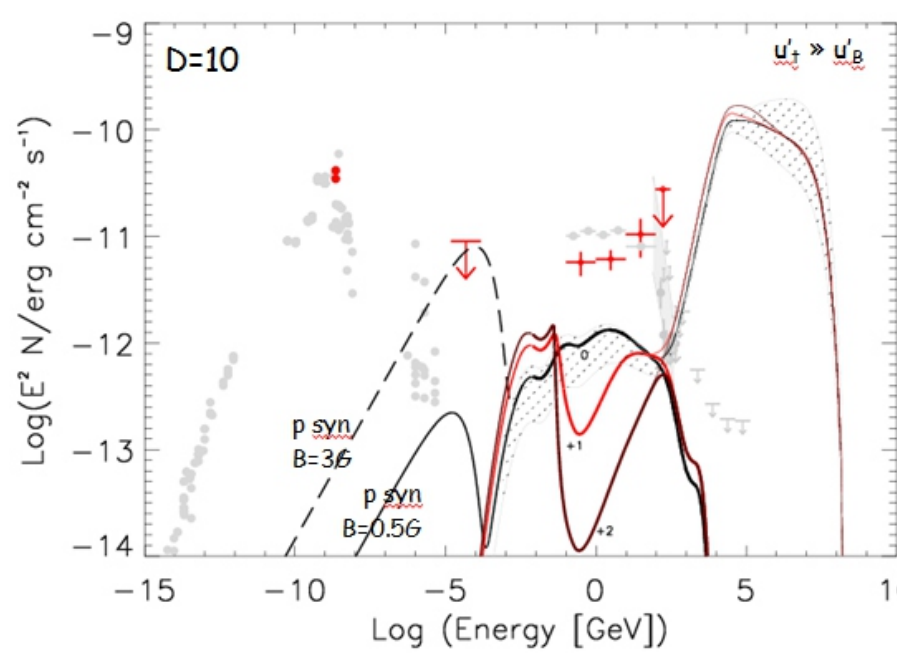
spectra arriving at Earth without (thin lines) and including absorption in the EBL (thick lines) for $\log \left(\tau_{\gamma \gamma \text {,max }}\right)=0,1,2$ as indicated, and $D=10$. The shaded areas represent the spread of the cascade spectra, for the case $\tau_{\gamma \gamma \text {,max }}=1$ as an example, propagated from the uncertainties of the observed neutrino spectrum. For the $\tau_{\gamma \gamma \text {,max }}=1$-case the corresponding proton synchrotron radiation component for two sub-equipartion $\left(u_{B}^{\prime} \ll u_{t}^{\prime}\right)$ field strengths are included. The red data points (ASAS-SN, SWIFT-BAT, Fermi-LAT) depict the quasi-simultaneous observations while the grey data points represent archival data (see [6] for details). This figure is adapted from [6].

In the other extreme case of $u_{t}^{\prime} \ll u_{B}^{\prime}$ synchrotron-supported cascades develop. Fig. 3 shows 
an example for the case of $u_{B}=10 u_{t}^{\prime}$ and $D=10$. In the optically thin case the standard synchrotron cooled spectra for a two-component (meson-decay and the lower energy Bethe-Heitler pairs) pair population is recovered. Changing the magnetization in the emission region has only a mild effect on the cascade fluxes, however, strongly alters the contribution from proton synchrotron radiation (see e.g., Fig. 4). Because the secondary pair distribution resulting from proton-photon interactions is fixed by the observed neutrino spectrum the corresponding synchrotron-supported cascade emission in the optically thin case is expected to be unaffected by a possible anisotropy of the collisions. In the optically thick case, $\tau_{\gamma \gamma} \gg 1$, the number of pairs, produced from synchrotron photons, increases dramatically in the cascade. The amount of reprocessing is given by $\tau_{\gamma \gamma}$, and the resulting cascade flux for a given $\tau_{\gamma \gamma}$ is therefore independent of whether it is produced by isotropic or anisotropic scatterings, as long as the contribution to the scattering rate per unit scattering angle is energy-independent. Similarly as for the optically thick Compton-supported cascades we note deep absorption troughs at $\mathrm{GeV}$ energies leading to the same implications for the association between the neutrino and $\mathrm{GeV}$-source. When compared to the quasi-simultaneous observed SED we note that deep optical-to-X-ray observations are able to provide sensitive constraints, in particular on the efficiency of neutrino production. Tab. 1 provides a summary of all constraints derived from the cascading calculations.

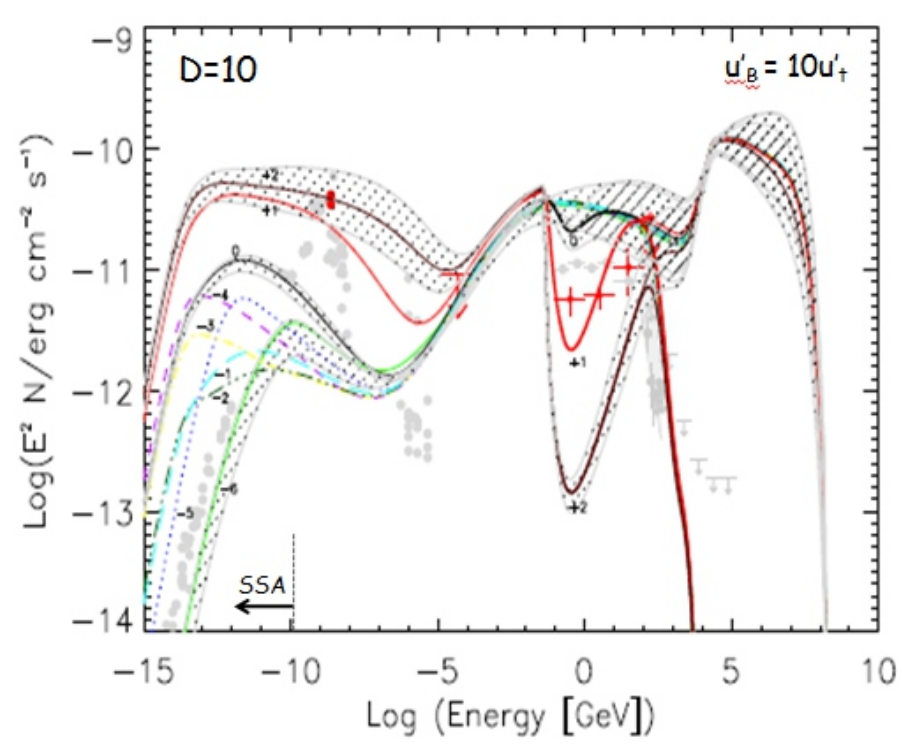

Figure 3: Synchrotron-supported cascade spectra arriving at Earth without (thin lines) and including absorption in the EBL (thick lines) for $u_{B}=10 u_{t}^{\prime}$ and $\log \left(\tau_{\gamma \gamma, \max }\right)=$ -6 (solid line), -5 (dotted line), -4 (short dashed line), -3 (dashed-dotted line), -2 (dashed-triple-dotted line), -1 (long dashed line), 0, 1, 2 (solid lines) as indicated, and $D=10$. The shaded areas represent the spread of the cascade spectra, for the cases $\tau_{\gamma \gamma \text {,max }}=10^{-6}, 1,100$ as examples, propagated from the uncertainties of the observed neutrino spectrum. Synchrotron-self absorption is not included in the calculated spectra while its spectral range of possible relevance is indicated in the figure. The data points are the same as shown in Fig. 2. This figure is adapted from [6].

\section{Implications}

Three constraints can now be combined to derive information about the environment where the neutrino production had occured in TXS 0506+056: First, the observed neutrino flux in the $30 \mathrm{TeV}-3 \mathrm{PeV}$ energy range provides limits on the combination of the cosmic ray and target photon field energy density given the Doppler factor. Second, the previously discussed comparison of the 


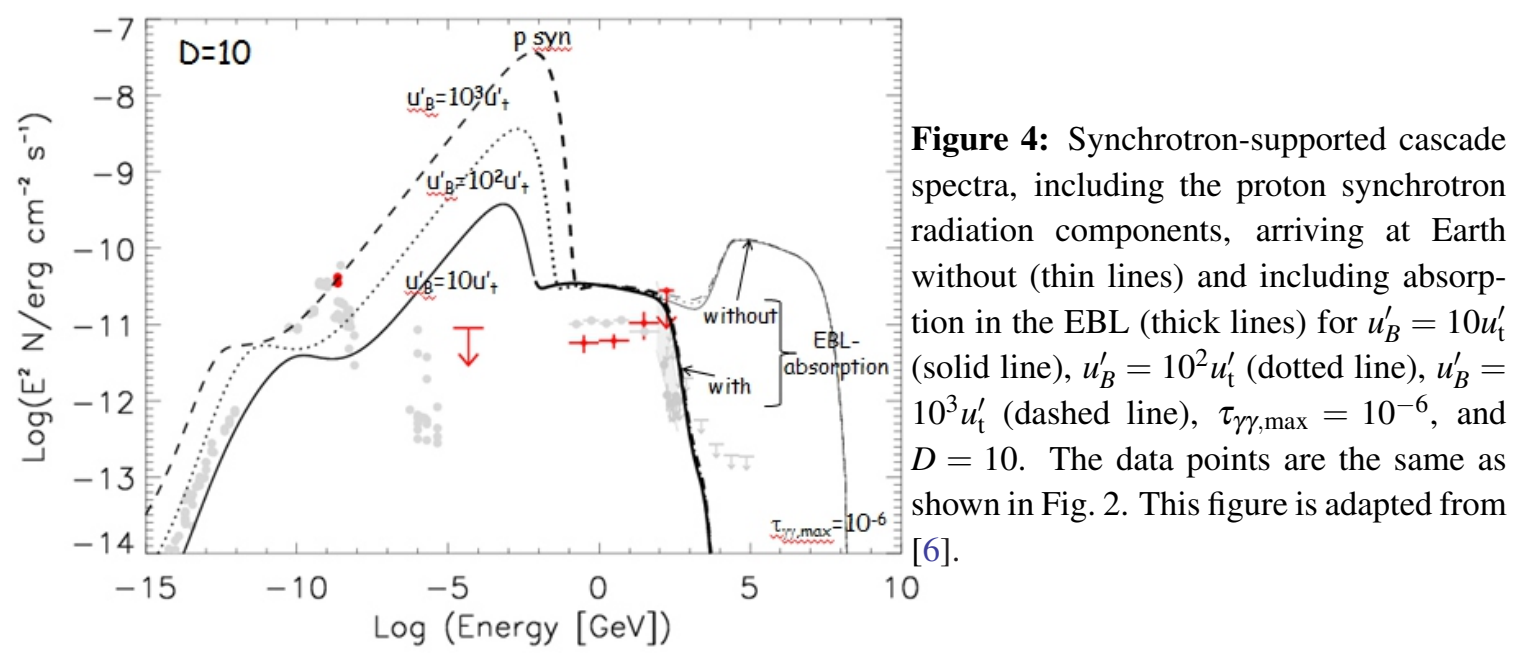

minimal cascade SEDs to the quasi-simultaneous broadband SED constrains the neutrino production efficiency ("cascading constraints"). Finally, the target photon field flux in the observer frame shall not exceed the observed flux in the relevant energy range. Here, the origin of the dominant target photon field plays a role. We distinguish two possible scenarios, which can be thought of as extreme, limiting cases: (a) a target photon field that is co-moving with the emission region, or (b) a stationary target photon field in the AGN rest frame. In case (a), in order to produce neutrinos at $30 \mathrm{TeV}-3 \mathrm{PeV}$ (observer frame) energies, the target photon energy range must lie in the hard X-ray regime, while in case (b), the external (stationary) target photon field is Doppler boosted into the blob frame, and therefore is observed as UV-to-soft X-rays.

From the archival hard X-ray flux of order $10^{-12} \mathrm{erg} \mathrm{cm}^{-2} \mathrm{~s}^{-1}$ in tandem with the observed neutrino flux the combination of co-moving target photon and cosmic ray energy density can be constrained for scenario (a). The corresponding minimal proton kinetic power $L_{p}$ for typical blazar Doppler factors lies at $\sim 10^{55} \mathrm{erg} / \mathrm{s}$ which exceeds the Eddington luminosity of the most massive black holes known by several orders of magnitude. Furthermore, this scenario predicts a proton synchrotron flux at keVs of $\sim 10^{4}$ times larger than the photo-pion induced emission (unless field strengths and/or Doppler factors extremely low; see Fig. 4). This is several orders of magnitude higher than any of the observed X-ray fluxes ever detected from this source. Based on these two arguments we rule out this scenario.

We next consider the case of a target photon field that is stationary in the AGN rest frame. Here we use the before discussed cascading results which gave opacity constraints only for an environment where synchrotron and/or Compton-synchrotron cascades operate (see Tab. 1). These opacity constraints correspond to a target photon field energy density that, transformed into the observer frame for case (b), results in a UV-to-soft X-ray flux that is several orders of magnitude higher than the archival data SED implies. On this basis we rule out environments where pair synchrotron emission (as compared to inverse Compton losses) can not be neglected any more.

What remains possible is a highly radiative environment where the magnetic field energy density is much lower than the target photon energy density in the jet frame. Here we have no cascading constraints as the minimum Compton cascade flux was always lower than the observed SED. The predicted proton synchrotron flux also appears on a level that does not violate observational lim- 


\begin{tabular}{c|c|l}
\hline Origin of target photon field & Further setup constraints & Results \\
\hline $\begin{array}{c}\text { All target photon fields } \\
\text { (cascading constraints) }\end{array}$ & $u_{t}^{\prime} \gg u_{B}^{\prime}$ & no constraints \\
& $u_{t}^{\prime} \leq u_{B}^{\prime}$ & $\begin{array}{l}\tau_{\gamma \gamma \text { max }} \sim(\text { a few }) 10 \text { for } D \leq 10 \\
\tau_{\gamma \gamma, \text { max }} \sim(\text { a few }) 10^{1 \ldots 2} \text { for } D \gg 10\end{array}$ \\
\hline Co-moving target photon field & & $\begin{array}{l}\text { ruled out: too high } L_{p} ; \\
p \text { syn overshoots X-ray archival flux }\end{array}$ \\
\hline Stationary target photon field & $u_{t}^{\prime} \leq u_{B}^{\prime}$ & $\begin{array}{l}\text { ruled out: target photon flux }(a) \\
\text { overshoots UV/soft X-ray archival flux } \\
\text { viable: target photon flux below UV/ } \\
\text { soft X-ray archival flux; } \\
\end{array}$ \\
& $u_{t}^{\prime} \gg u_{B}^{\prime}$ acceptable for sufficiently large $D$ \\
\hline
\end{tabular}

Table 1: Summary of tested scenarios and results.

(a) Includes cascading constraints.

its implied by archival data (being of the order of $10^{-12} \mathrm{erg} \mathrm{cm}^{-2} \mathrm{~s}^{-1}$ ). Further the corresponding required proton power lies near the Eddington limit for sufficiently large Doppler factors $D \sim 50$. Hence this scenario seems possible. In this case we predict pair production opacities in this region below unity for typical blazar environments. This in turn gives a limit of the neutrino production efficiency of order $10^{-3}$ for the photomeson production opacity. Hence TXS $0506+056$ must have been an inefficient neutrino producer during the neutrino flare. This is in disagreement with what IceCube data suggest [2]. Moreover, from the limit on the pair production opacity we can infer the level of the cascade flux at $\mathrm{GeV}$ energies which is significantly below the observed LAT-flux. Further radiation processes which are unlikely proton-initiated are therefore required to explain the observed $\gamma$-ray SED. The bulk of the LAT-photons and IceCube-neutrinos received during the neutrino flare can not have been initiated by the same process. Similar conclusions has been reached by [8].

The detailed calculations supporting the aforementioned argumentations can be found in [6].

\section{Summary}

We presented a procedure to derive constraints on the broadband photon SED for a given neutrino spectrum in the framework of photo-hadronically produced neutrinos in jetted AGN. It is based on electromagnetic cascading, induced by the photons and electron-positrons that were coproduced with the neutrinos on a minimal target photon field. This procedure can accommodate any origin for the target photon field. We discussed in particular the case of external target photon fields that is thought to be relevant for FSRQs, and applied it to the 2014-15 neutrino flare spectrum of the FSRQ TXS 0506+056. We then combined the derived limits on the $\gamma \gamma$ opacity from the cascade SEDs and co-moving neutrino luminosity of TXS 0506+056 with direct observational constraints from its MWL SED in the expected energy range of the required minimum target photon field. The so derived constraints allow us to characterize the environment of the neutrino-production region as

- a highly radiative site where $u_{B}^{\prime} \ll u_{t}^{\prime}$, 
- the dominant target photon field for particle/photon-photon interactions is external to the jet,

- the proton kinetic power is of order Eddington luminosity for sufficiently large Doppler factors,

- the detected GeV-flux can not originate from the neutrino-production site (which in turn questions a correlation between neutrino- and $\mathrm{GeV} / \mathrm{TeV}$ gamma-ray source activity),

- the detected neutrino flux is produced at rather low rates during the "neutrino flare".

\section{Acknowledgements}

A.R. acknowledges financial support from the Austrian Science Fund (FWF), project I 3452-N27. The Fermi-LAT Collaboration acknowledges support for LAT development, operation and data analysis from NASA and DOE (United States), CEA/Irfu and IN2P3/CNRS (France), ASI and INFN (Italy), MEXT, KEK, and JAXA (Japan), and the K.A. Wallenberg Foundation, the Swedish Research Council and the National Space Board (Sweden). Science analysis support in the operations phase from INAF (Italy) and CNES (France) is also gratefully acknowledged. This work performed in part under DOE Contract DE-AC02-76SF00515. The work of M. Böttcher is supported by the South African Research Chairs Initiative (grant no. 64789) of the Department of Science and Technology and the National Research Foundation ${ }^{1}$ of South Africa.

\section{References}

[1] M.G. Aartsen, et al. (IceCube Collaboration), Multimessenger observations of a flaring blazar coincident with high-energy neutrino IceCube-170922A, Science 361 (2018) eaat1378.

[2] M.G. Aartsen, et al. (IceCube Collaboration), Neutrino emission from the direction of the blazar TXS 0506+056 prior to the IceCube-170922A alert, Science 361 (2018) 147-151.

[2] Garrappa, S., et al. (Fermi-LAT collaboration, IceCube Collaboration), Investigation of two Fermi-LAT gamma-ray blazars coincident with high-energy neutrinos detected by IceCube, ApJ (2019), accepted [arXiv:1901.10806].

[3] M. Ajello, R. W. Romani, D. Gasparrini, et al. The Cosmic Evolution of Fermi BL Lacertae Objects, ApJ 780 (2014) 73 [arXiv: 1310.0006 ].

[4] S. Paiano, R. Falomo, A. Treves \& R. Scarpa, The redshift of the BL Lac object TXS 0506+056, ApJ 854 (2018) L32 [arXiv: 1802 . 01939].

[5] P. Padovani, F. Oikonomou, M. Petropoulou, P. Giommi, E. Resconi, TXS 0506+056, the first cosmic neutrino source, is not a BL Lac, MNRAS 484 (2019) L104 [arXiv: 1901.06998 ].

[6] A. Reimer, M. Böttcher, S. Buson, Cascading constraints from neutrino emitting blazars: The case of TXS 0506+056, ApJ (2019), accepted [arXiv: 1812 . 05654].

[7] A. Franceschini \& G. Rodighiero, The extragalactic background light revisited and the cosmic photon-photon opacity, Astronomy \& Astrophysics 603 (2017) A34 [arXiv: 1705.10256 ].

[8] X. Rodrigues, S. Gao, A. Fedynitch, A. Palladino, W. Winter, Leptohadronic blazar models applied to the 2014-15 flare of TXS 0506+056, ApJL 874 (2019) A34 [arXiv: 1812 . 05939].

\footnotetext{
${ }^{1}$ Any opinion, finding and conclusion or recommendation expressed in this material is that of the authors and the NRF does not accept any liability in this regard.
} 